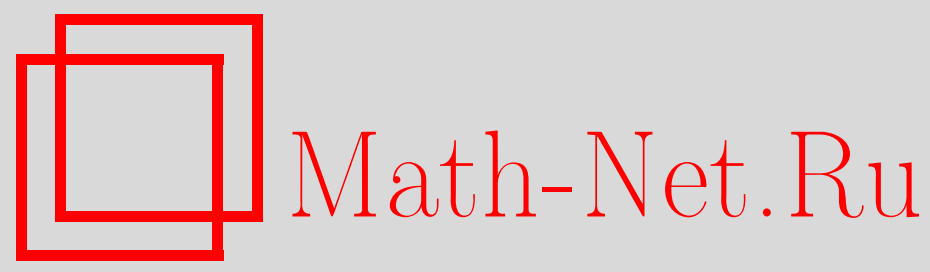

F. M. Malyshev, A. E. Trishin, Линейный и разностный криптоанализ: другая точка зрения, Матем. вопр. криптогр., 2020, том 11, выпуск 2, 83-98

DOI: https://doi.org/10.4213/mvk323

Использование Общероссийского математического портала Math-Net.Ru подразумевает, что вы прочитали и согласны с пользовательским соглашением http://www . mathnet.ru/rus/agreement

Параметры загрузки:

IP : 54.198 .187 .58

26 апреля 2023 г., $14: 58: 23$

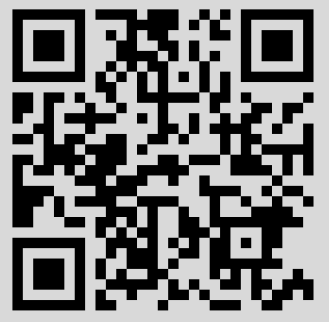




\title{
Linear and differential cryptanalysis: Another viewpoint
}

\author{
F. M. Malyshev ${ }^{1}$, A. E. Trishin ${ }^{2}$ \\ ${ }^{1}$ Steklov Mathematical Institute of RAS, Moscow \\ ${ }^{2}$ Certification Research Center LLC, Moscow
}

Получено 25.XI.2019

\begin{abstract}
Theorems on the exact values of advantages for linear and differential cryptanalysis are proved. The example of universal functional scheme illustrates a wide range of possible errors when the usual methods of estimation the advantages of probabilistic relations are used. It is argued that the probabilistic relations should be constructed for fixed cipher keys separately. The duality between the linear and the differential cryptanalysis is rigorously formulated. The degree of diffusion in linear medium is introduced; it is shown that its maximization should be one of the basic principles of cipher design. This is a quantitative measure of Shannon's qualitative principle that ciphers should realize transforms with high diffusion.
\end{abstract}

Keywords: linear cryptanalysis, differential cryptanalysis, linear medium, block ciphers

\section{Линейный и разностный криптоанализ: другая точка зрения Ф. М. Малышев ${ }^{1}$, А. Е. Тришин ${ }^{2}$ \\ ${ }^{1}$ Математический институт им.В.А.Стеклова Российской академии наук, Москва, \\ ${ }^{2}$ ООО «Центр сертификачионных исследований», Москва}

Аннотация. Доказаны теоремы о точных значениях линейной и разностной характеристик. Приведен пример универсальной функциональной схемы, который показывает, что обычные методы оценки характеристик вероятностных соотношений могут приводить к значительным ошибкам. Отстаивается мнение о том, что вероятностные соотношения нужно строить для фиксированных криптографических ключей. Математически строго сформулирована двойственность линейного и разностного методов. Введены показатели рассеивания линейной среды, максимизация которых является одним из базовых принципов современного криптографического синтеза. Они 
формализуют качественное требование К. Шеннона к шифрам как преобразованиям, обеспечивающим хорошее рассеивание.

Ключевые слова: линейный метод, разностный метод, линейная среда, блочный шифр

\section{Introduction}

The point of view on linear and differential cryptanalysis presented here was developed in parallel with their other numerous representations in cryptographic literature. These methods should be called local linear and local differential cryptanalysis more correctly.

This paper is devoted to a technique for constructing a probabilistic linear and differential relations for functions $F: V_{N} \rightarrow V_{M}$ defined by functional schemes. Here $V_{N}=G F(2)^{N}, V_{M}=G F(2)^{M}$ are $N$ - and $M$-dimensional coordinate spaces over the field $G F(2)$. In the next section some definitions related to functional schemes are given.

Depending on the goals of the cryptanalyst, the mapping $F$ may be either the whole cipher transformation parameterized by the key, or a separate fragment of the whole cipher transformation, which may also be (and this is most often) parameterized by the key or by part of it.

Let $V_{N}^{*}$ denote the set of all Boolean column vectors of length $N$. The non-strict equation $a L^{\prime} \simeq b L^{\prime \prime}, a \in V_{N}, b=F(a) \in V_{M}$, is called a probabilistic linear relation defined by column vectors $L^{\prime} \in V_{N}^{*}, L^{\prime \prime} \in V_{M}^{*}$. The measure of its strictness is defined as $\delta_{L^{\prime}, L^{\prime \prime}}=\delta_{L^{\prime}, L^{\prime \prime}}^{F}=2 \mathbf{P}\left\{a L^{\prime}=b L^{\prime \prime}\right\}-1$ under the condition that $a$ is a random vector with the uniform probabilistic distribution on $V_{N}$ and $b=F(a) \in V_{M}$. The value $\delta_{L^{\prime}, L^{\prime \prime}}$ is called an advantage of a probabilistic linear relation (or linear advantage).

Let $D^{\prime} \in V_{N}, D^{\prime \prime} \in V_{M}$ be any fixed vectors, $a^{(1)}, a^{(2)} \in V_{N}, b^{(1)}$, $b^{(2)} \in V_{M}$ be vectors such that $b^{(1)}=F\left(a^{(1)}\right), b^{(2)}=F\left(a^{(2)}\right)$. The non-strict implication «if $a^{(1)}+a^{(2)}=D^{\prime}$, then $b^{(1)}+b^{(2)}=D^{\prime \prime} \gg$ is called a probabilistic differential relation and denoted by $\left(D^{\prime}, D^{\prime \prime}\right)$. A measure of strictness of this implication defined as $p_{D^{\prime}, D^{\prime \prime}}=p_{D^{\prime}, D^{\prime \prime}}^{F}=\mathbf{P}\left\{F\left(a+D^{\prime}\right)+F(a)=D^{\prime \prime}\right\}$ is called an advantage of a differential relation (or differential advantage). Here $a$ is also a random vector with the uniform probabilistic distribution on $V_{N}$.

Probabilistic linear and differential relations are used, for example, in attacks on cryptographic keys of ciphers usually defined by functional schemes. In such case the function $F: V_{N} \rightarrow V_{M}$ is a part of the full cipher. This part depends on the key $X$ (the function $F$ is parameterized by the 
key $X)$. The argument $a \in V_{N}$ of the mapping $F$ are generated from plain text and the key $Z$. The problem is to find the key $Z$, not $X$.

The theoretical results of this paper were obtained by the first author on the basis of many linear probability relations found by him for specific stream and block ciphers. The second author had performed nontrivial practical applications of these theoretical results to some block ciphers which supports their interpretation.

Terminology used in this paper differs from the generally accepted one. For example, we use the term probabilistic relations instead of approximations or differentials, the term chains of conformal local relations instead of characteristics, the term degree of diffusion instead of the number of active S-boxes. We use the term advantage (linear or differential) for the measure of strictness of probabilistic relations.

\section{The linear medium of a functional scheme}

The function $F: V_{N} \rightarrow V_{M}, V_{N} \ni a \mapsto b=F(a) \in V_{M}$, defined by a cipher (or by a part of it) with a fixed key $X$ is called a cipher transformation. It is specified using the functional scheme $\mathcal{F}$, i.e. the set of linear and nonlinear mappings. These mappings may be applied one after another and be realized by a computer program without cycles.

Let the nonlinear mappings $f_{i}: V_{n_{i}} \rightarrow V_{m_{i}}, x_{i} \mapsto y_{i}=f_{i}\left(x_{i}\right), i=$ $1, \ldots, k$, be performed in this program in a certain order. These mappings are called functional elements. As a rule, functional elements are nonlinear, but linear functional elements are also allowed. The argument $x_{i} \in V_{n_{i}}$ of the function $f_{i}$ is the result of sequential application of some previous operations to $a \in V_{N}$. The linear operations are used between $f_{i}, i=1, \ldots, k$. Any linear operation may be realized by the additions modulo 2 and reproduction nodes with several outputs $x \mapsto(x, \ldots, x)$. When considering a block cipher the parameter $k$ is not the same as the number of iterations (rounds). Typically one iteration consists of several mappings $f_{i}$.

We do not consider linear operations of a cipher separately. Instead for some Boolean $m_{i} \times n_{j}$ matrices $c_{i j}, i=0,1, \ldots, k, j=1, \ldots, k, k+1$, $m_{0}=N, n_{k+1}=M$, we can write $x_{j}=a c_{0 j}+\sum_{i=1}^{j-1} y_{i} c_{i j}, j=1, \ldots, k$, $b=a c_{0, k+1}+\sum_{i=1}^{k} y_{i} c_{i, k+1}$. Let $c_{i j}=0$ if $i \geqslant j$. Then it's possible to construct a matrix $C=C_{\mathcal{F}}$ of size $\left(N+\sum_{i=1}^{k} m_{i}\right) \times\left(\sum_{i=1}^{k} n_{i}+M\right)$ with blocks $c_{i j}$ such that

$$
\left(a, y_{1}, \ldots, y_{k}\right) C=\left(x_{1}, \ldots, x_{k}, b\right) .
$$


The matrix $C$ is said to be a matrix of linear medium of the functional scheme $\mathcal{F}$. It integrates all linear operations of the cipher transformation. The linear medium is denoted by the same letter $C$ and is also a functional scheme. It is obtained from the functional scheme $\mathcal{F}$ by deletion of all functional elements $f_{i}, i=1, \ldots, k$. As a result all $n_{i}$ Boolean inputs of any functional element $f_{i}$ becomes the outputs of the linear medium $C$ and all $m_{i}$ Boolean outputs of this element becomes the inputs of the linear medium $C$. The linear medium $C$ determines a linear mapping $C: V_{N+\sum_{i=1}^{k} m_{i}} \rightarrow V_{\sum_{i=1}^{k} n_{i}+M}$ in accordance with (1).

Thus the functional scheme $\mathcal{F}$ is represented by the linear medium $C$ into which the nonlinear elements $f_{i}: V_{n_{i}} \rightarrow V_{m_{i}}, i=1, \ldots, k$, are embedded. As a result we are able to study separately properties of the linear medium $C$ of the cipher transformation $F$ and properties of its nonlinear part consisting of separate functions $f_{i}, i=1, \ldots, k$.

The representation of the cipher transformation by functional scheme $\mathcal{F}$ is not uniquely defined. The cryptanalyst himself chooses a certain functional scheme $\mathcal{F}$ determining the cipher transformation $F$. If he chooses local elements $f_{i}, i=1, \ldots, k$, too complex, then the analysis of each element will be difficult, but this will reduce their number and simplify the linear medium. On the other hand, if he chooses elements $f_{i}$ too simple (up to the Sheffer functions), it will increase their number and will complicate the linear medium.

\section{Chains of conformal local linear relations}

The main problem of linear cryptanalysis is to construct a pair of column vectors $L^{\prime} \in V_{N}^{*}, L^{\prime \prime} \in V_{M}^{*} \backslash\{0\}$ such that the absolute value of the advantage $\delta_{L^{\prime}, L^{\prime \prime}}=\delta_{L^{\prime}, L^{\prime \prime}}^{F}=2 \mathbf{P}\left\{a L^{\prime}=b L^{\prime \prime}\right\}-1$ of linear relation $a L^{\prime} \simeq b L^{\prime \prime}$ is as high as possible. Here $b=F(a)$ and the vector $a$ has the uniform probabilistic distribution on $V_{N}$.

The probabilistic linear relation $a L^{\prime} \simeq b L^{\prime \prime}$ or, what is the same, $a L^{\prime}+$ $b L^{\prime \prime} \simeq 0$ may be obtained by formal addition modulo 2 of appropriate local probabilistic linear relations

$$
x_{i} l_{i}^{\prime}+y_{i} l_{i}^{\prime \prime} \simeq 0, \quad l_{i}^{\prime} \in V_{n_{i}}^{*}, l_{i}^{\prime \prime} \in V_{m_{i}}^{*}, \quad y_{i}=f_{i}\left(x_{i}\right), \quad i=1, \ldots, k,
$$

characterized by linear advantages $\delta_{i}=\delta_{l_{i}^{\prime}, l_{i}^{\prime \prime}}^{f_{i}}=2 \mathbf{P}\left\{x_{i} l_{i}^{\prime}=y_{i} l_{i}^{\prime \prime}\right\}-1$; each advantage $\delta_{i}$ is computed under condition that each vector $x_{i}$ has the uniform probabilistic distribution on $V_{n_{i}}$. Note that there are cipher transformations $F$ for which the distribution of $x \in V_{n_{i}}$ is not uniform for any distribution of random $a \in V_{N}$. 
The set $\mathfrak{L}=\left(\left(l_{i}^{\prime}, l_{i}^{\prime \prime}\right), i=1, \ldots, k\right)$ is called conformal if for some $L^{\prime} \in V_{N}^{*}$, $L^{\prime \prime} \in V_{M}^{*}$ we have the equality $\sum_{i=1}^{k}\left(x_{i} l_{i}^{\prime}+y_{i} l_{i}^{\prime \prime}\right)=a L^{\prime}+b L^{\prime \prime}$, or the following chain of equalities

$$
\begin{aligned}
& 0=\sum_{i=1}^{k}\left(x_{i} l_{i}^{\prime}+y_{i} l_{i}^{\prime \prime}\right)+a L^{\prime}+b L^{\prime \prime}=x l^{\prime}+y l^{\prime \prime}+a L^{\prime}+b L^{\prime \prime}=(a, y)\left(\begin{array}{c}
L^{\prime} \\
l^{\prime \prime}
\end{array}\right) \\
& +(x, b)\left(\begin{array}{c}
l^{\prime} \\
L^{\prime \prime}
\end{array}\right)=(a, y)\left(\begin{array}{c}
L^{\prime} \\
l^{\prime \prime}
\end{array}\right)+(a, y) C\left(\begin{array}{c}
l^{\prime} \\
L^{\prime \prime}
\end{array}\right)=(a, y)\left[\left(\begin{array}{c}
L^{\prime} \\
l^{\prime \prime}
\end{array}\right)+C\left(\begin{array}{c}
l^{\prime} \\
L^{\prime \prime}
\end{array}\right)\right]
\end{aligned}
$$

with respect to any variables $x=\left(x_{1}, \ldots, x_{k}\right), y=\left(y_{1}, \ldots, y_{k}\right)$ such that $x_{i}, y_{i}, i=1, \ldots, k$, are connected with vectors $a$ and $b$ only by linear relation (1) and should not satisfy equations $y_{i}=f_{i}\left(x_{i}\right)$; further, $l^{\prime} \in V_{\sum_{i=1}^{*} n_{i}}$ is a concatenation of vectors $l_{i}^{\prime}$, similarly $l^{\prime \prime} \in V_{\sum_{i=1}^{*} m_{i}}$ is a concatenation of vectors $l_{i}^{\prime \prime}, i=1, \ldots, k$. Thus the set $\mathfrak{L}=\left(\left(l_{i}^{\prime}, l_{i}^{\prime \prime}\right), i=1, \ldots, k\right)$ is conformal iff

$$
C\left(\begin{array}{c}
l^{\prime} \\
L^{\prime \prime}
\end{array}\right)=\left(\begin{array}{l}
L^{\prime} \\
l^{\prime \prime}
\end{array}\right)
$$

for some $L^{\prime}=L_{\mathfrak{L}}^{\prime} \in V_{N}^{*}$ and $L^{\prime \prime}=L_{\mathfrak{L}}^{\prime \prime} \in V_{M}^{*}$.

We can consider a product $\tilde{\delta}=\delta_{1} \cdot \ldots \cdot \delta_{k}, \delta_{i}=\delta_{l_{i}^{\prime}, l_{i}^{\prime \prime}}^{f_{i}}, i=1, \ldots, k$, as a rough approximation (approximate value) for the linear advantage $\delta_{L^{\prime}, L^{\prime \prime}}$. Such approximation may be MOTIVATED by the following note. The equality $\delta_{L^{\prime}, L^{\prime \prime}}=\tilde{\delta}$ would be true if all events $\left\{x_{i} l_{i}^{\prime}=y_{i} l_{i}^{\prime \prime}\right\}, i=1, \ldots, k$, were statistically independent and corresponding equalities $\delta_{i}=2 \mathbf{P}\left\{x_{i} l_{i}^{\prime}=\right.$ $\left.y_{i} l_{i}^{\prime \prime}\right\}-1$ for equiprobable $a \in V_{N}$ were satisfied. In fact the exact fulfillment of such conditions seems almost impossible.

Let $\mathfrak{W}\left(L^{\prime}, L^{\prime \prime}\right)=\mathfrak{W}_{\mathcal{F}}\left(L^{\prime}, L^{\prime \prime}\right)$ be the set of all solutions $\mathfrak{L}=\left(\left(l_{i}^{\prime}, l_{i}^{\prime \prime}\right), i=\right.$ $1, \ldots, k$ ) of system (2) for given $L^{\prime} \in V_{N}^{*}, L^{\prime \prime} \in V_{M}^{*}$ (the vectors $L^{\prime}, L^{\prime \prime}$ are called boundary conditions). Let $\mathfrak{W}=\bigcup_{L^{\prime} \in V_{N}^{*}, L^{\prime \prime} \in V_{M}^{*}} \mathfrak{W}\left(L^{\prime}, L^{\prime \prime}\right)$ and $\mathfrak{W}^{(0)}=\left\{\mathfrak{L} \in \mathfrak{W} \mid l_{i}^{\prime \prime}=0 \Rightarrow l_{i}^{\prime}=0 \forall i=1, \ldots, k\right\}$. If $\mathfrak{L} \in \mathfrak{W} \backslash \mathfrak{W}^{(0)}$ then $\tilde{\delta}_{\mathfrak{L}}=0$, where $\tilde{\delta}_{\mathfrak{L}}=\prod_{i=1}^{k} \delta_{l_{i}^{\prime}, l_{i}^{\prime \prime}}^{f_{i}}$. Indeed, if $l_{i}^{\prime \prime}=0, l_{i}^{\prime} \neq 0$, then $\delta_{l_{i}^{\prime}, l_{i}^{\prime \prime}}^{f_{i}}=0$, $i=1, \ldots, k$. To construct the required linear relation we have to find a set $\mathfrak{L} \in \mathfrak{W}^{(0)}$ for which the value $\left|\tilde{\delta}_{\mathfrak{L}}\right|$ is as high as possible, so the factors equal to \pm 1 are preferable in the product $\tilde{\delta}_{\mathfrak{L}}$. These factors may appear if $l_{i}^{\prime \prime}=0$. Suppose that $\mathfrak{L} \in \mathfrak{W}^{(0)}$. Let $\theta_{\mathfrak{L}}=\left|\left\{i \in\{1, \ldots, k\} \mid l_{i}^{\prime \prime} \neq 0\right\}\right|$ be the number of factors in product $\tilde{\delta}_{\mathfrak{L}}$ possibly not equal to one. To maximize $\left|\tilde{\delta}_{\mathfrak{L}}\right|$ sometimes it is reasonable to find the number $\theta_{C}=\min _{\mathfrak{L} \in \mathfrak{W}^{(0)} \backslash\{0\}} \theta_{\mathfrak{L}}$ called a degree of diffusion of the linear medium $C$ with respect to linear 
cryptanalysis. Any set $\mathfrak{L} \in \mathfrak{W}^{(0)}$ is called a chain of conformal local linear relations if it includes only nontrivial (when $l_{i}^{\prime \prime} \neq 0$ ) local linear relations $x_{i} l_{i}^{\prime} \simeq y_{i} l_{i}^{\prime \prime}, i=1, \ldots, k$.

The technique described in this section may be applied to the search for multidimensional ( $s$-dimensional) linear relations [1]. In this case it is necessary to consider matrices with $s$ columns instead of column vectors $L^{\prime}, L^{\prime \prime}, l_{i}^{\prime}, l_{i}^{\prime \prime}, i=1, \ldots, k$.

\section{Chains of conformal local differential relations}

If we consider equations (1) for two inputs $a^{(1)}, a^{(2)} \in V_{N}$ of the functional scheme and subtract one from the other, we will obtain

$$
\left(D^{\prime}, d_{1}^{\prime \prime}, \ldots, d_{k}^{\prime \prime}\right) C=\left(d_{1}^{\prime}, \ldots, d_{k}^{\prime}, D^{\prime \prime}\right)
$$

where $D^{\prime}=a^{(1)}+a^{(2)}, D^{\prime \prime}=b^{(1)}+b^{(2)}, d_{i}^{\prime \prime}=y_{i}^{(1)}+y_{i}^{(2)}, d_{i}^{\prime}=x_{i}^{(1)}+x_{i}^{(2)}$, $i=1, \ldots, k$. Here $b^{(1)}=F\left(a^{(1)}\right), b^{(2)}=F\left(a^{(2)}\right)$ and $x_{i}^{(1)}, x_{i}^{(2)}$ are arguments of the function $f_{i}$ if $a^{(1)}$ and $a^{(2)}$ are the inputs of the functional scheme, $y_{i}^{(1)}=f_{i}\left(x_{i}^{(1)}\right), y_{i}^{(2)}=f_{i}\left(x_{i}^{(2)}\right), i=1, \ldots, k$.

The main problem of differential cryptanalysis is to construct a pair of vectors $D^{\prime} \in V_{N} \backslash\{0\}, D^{\prime \prime} \in V_{M}$ such that the differential advantage $p_{D^{\prime}, D^{\prime \prime}}=p_{D^{\prime}, D^{\prime \prime}}^{F}=\mathbf{P}\left\{F\left(a+D^{\prime}\right)+F(a)=D^{\prime \prime}\right\}$ of the non-strict implication $a^{(1)}+a^{(2)}=D^{\prime} \Rightarrow b^{(1)}+b^{(2)}=D^{\prime \prime}$ is as high as possible.

Let $D^{\prime} \in V_{N}, D^{\prime \prime} \in V_{M}, d_{i}^{\prime} \in V_{n_{i}}, d_{i}^{\prime \prime} \in V_{m_{i}}, i=1, \ldots, k$, be an arbitrary set of vectors satisfying conformity condition (3). Since $C$ is a block upper triangular matrix, then from implications $x_{i}^{(1)}+x_{i}^{(2)}=d_{i}^{\prime} \Rightarrow y_{i}^{(1)}+y_{i}^{(2)}=d_{i}^{\prime \prime}$ for all $i=1, \ldots, k$ it follows that $a^{(1)}+a^{(2)}=D^{\prime} \Rightarrow b^{(1)}+b^{(2)}=D^{\prime \prime}$.

Let the set of local differences $\mathfrak{D}=\left(\left(d_{i}^{\prime}, d_{i}^{\prime \prime}\right), i=1, \ldots, k\right), d_{i}^{\prime} \in V_{n_{i}}$, $d_{i}^{\prime \prime} \in V_{m_{i}}$, satisfy the condition (3) for some boundary vectors $D^{\prime}=D_{\mathfrak{D}}^{\prime} \in$ $V_{N}, D^{\prime \prime}=D_{\mathfrak{D}}^{\prime \prime} \in V_{M}$. Consider a product $\tilde{p}_{\mathfrak{D}}=\prod_{i=1}^{k} p_{d_{i}^{\prime}, d_{i}^{\prime \prime}}^{f_{i}}$ of advantages $p_{d_{i}^{\prime}, d_{i}^{\prime \prime}}^{f_{i}}=\mathbf{P}\left\{f_{i}\left(x_{i}^{(1)}+d_{i}^{\prime}\right)+f_{i}\left(x_{i}^{(1)}\right)=d_{i}^{\prime \prime}\right\}$ of local differential relations $\left(d_{i}^{\prime}, d_{i}^{\prime \prime}\right)$, where we suppose that each vector $x_{i}^{(1)}$ has the uniform probabilistic distribution on $V_{n_{i}}$. Note that there are cipher transformations $F$ for which the distribution of $x \in V_{n_{i}}$ is not uniform for any distribution of random $a \in V_{N}$.

We can consider the product $\tilde{p}_{\mathfrak{D}}$ as a rough approximation for the advantage $p_{D^{\prime}, D^{\prime \prime}}^{F}$ of the differential relation $\left(D^{\prime}, D^{\prime \prime}\right) \in V_{N} \times V_{M}$. Such approximation may be MOTIVATED by the following note. If under condition that 
random input $a^{(1)}$ is uniformly distributed on $V_{N}$ and $a^{(2)}=a^{(1)}+D^{\prime}$, all random variables $x_{i}^{(1)} \in V_{n_{i}}, i=1, \ldots, k$, were independent and uniformly distributed and events $\left\{y_{i}^{(1)}+y_{i}^{(2)}=d_{i}^{\prime \prime}\right\}, i=1, \ldots, k$, were statistically independent, then using (3) we have

$$
\begin{gathered}
p_{D^{\prime}, D^{\prime \prime}}^{F} \geqslant \mathbf{P}\left\{y_{i}^{(1)}+y_{i}^{(2)}=d_{i}^{\prime \prime}, i=1, \ldots, k\right\} \\
=\prod_{i=1}^{k} \mathbf{P}\left\{f_{i}\left(x_{i}^{(1)}\right)+f_{i}\left(x_{i}^{(2)}\right)=d_{i}^{\prime \prime}\right\} \\
=\prod_{i=1}^{k} \mathbf{P}\left\{f_{i}\left(x_{i}^{(1)}\right)+f_{i}\left(x_{i}^{(1)}+d_{i}^{\prime}\right)=d_{i}^{\prime \prime}\right\}=\tilde{p}_{\mathfrak{D}} .
\end{gathered}
$$

In fact the exact fulfillment of hypotheses used in this estimate seems almost impossible.

Let $W\left(D^{\prime}, D^{\prime \prime}\right)=W_{\mathcal{F}}\left(D^{\prime}, D^{\prime \prime}\right)$ be the set of all solutions of the system (3) with given boundary differences $D^{\prime} \in V_{N}, D^{\prime \prime} \in V_{M}$ with respect to the set of local differences $\mathfrak{D}=\left(\left(d_{i}^{\prime}, d_{i}^{\prime \prime}\right), i=1, \ldots, k\right)$. Let $W=\bigcup_{D^{\prime} \in V_{N}, D^{\prime \prime} \in V_{M}} W\left(D^{\prime}, D^{\prime \prime}\right)$ and $W^{(0)}=\left\{\mathfrak{D} \in W \mid d_{i}^{\prime}=0 \Rightarrow d_{i}^{\prime \prime}=\right.$ $0 \forall i=1, \ldots, k\}$. If $\mathfrak{D} \in W \backslash W^{(0)}$, then $\tilde{p}_{\mathfrak{D}}=0$. Indeed, if $d_{i}^{\prime}=0, d_{i}^{\prime \prime} \neq 0$, then $p_{d_{i}^{\prime}, d_{i}^{\prime \prime}}^{f_{i}}=0, i=1, \ldots, k$. To construct the required differential relation we have to find a set $\mathfrak{D} \in W^{(0)}$ such that the value $\tilde{p}_{\mathfrak{D}}=\prod_{i=1}^{k} p_{d_{i}^{\prime}, d_{i}^{\prime \prime}}^{f_{i}}$ is as high as possible, so the factors equal to one are preferable in this product. These factors may appear if $d_{i}^{\prime}=0$. For any $\mathfrak{D} \in W^{(0)}$ let $\theta_{\mathfrak{D}}^{\prime}=\left|\left\{i \in\{1, \ldots, k\} \mid d_{i}^{\prime} \neq 0\right\}\right|$ be the number of factors in product $\tilde{p}_{\mathfrak{D}}$ possibly not equal to one. To maximize $\tilde{p}_{\mathfrak{D}}$ sometimes it is reasonable to find a number $\theta_{C}^{\prime}=\min _{\mathfrak{D} \in \mathrm{W}^{(0)} \backslash\{0\}} \theta_{\mathfrak{D}}^{\prime}$ called a degree of diffusion of the linear medium $C$ with respect to differential cryptanalysis. Any set $\mathfrak{D} \in W^{(0)}$ is called a chain of conformal local differential relations if it includes only nontrivial $\left(d_{i}^{\prime} \neq 0\right)$ local differential relations among $\left(d_{i}^{\prime}, d_{i}^{\prime \prime}\right), i=1, \ldots, k$.

\section{Theorems on exact values of linear and differential advantages}

Theorem 1. If $L^{\prime} \in V_{N}^{*}, L^{\prime \prime} \in V_{M}^{*}$, then $\delta_{L^{\prime}, L^{\prime \prime}}^{F}=\sum_{\mathfrak{L} \in \mathfrak{W}\left(L^{\prime}, L^{\prime \prime}\right)} \tilde{\delta}_{\mathfrak{L}}$. 
Theorem 2. If $D^{\prime} \in V_{N}, D^{\prime \prime} \in V_{M}$, then

$$
\begin{aligned}
& p_{D^{\prime}, D^{\prime \prime}}^{F} \\
& =\sum_{\mathfrak{D} \in W\left(D^{\prime}, D^{\prime \prime}\right)} \tilde{p}_{\mathcal{D}}+\frac{1}{2^{M}} \sum_{\left(L^{\prime}, L^{\prime \prime}\right) \in V_{N}^{*} \times V_{M}^{*}}(-1)^{D^{\prime} L^{\prime}+D^{\prime \prime} L^{\prime \prime}} \sum_{\substack{\mathfrak{s}_{1}, \mathfrak{L}_{2} \in \mathfrak{2} \mathcal{Y}\left(L^{\prime}, L^{\prime \prime}\right): \\
\mathfrak{L}_{1} \neq \mathcal{L}_{2}}} \tilde{\delta}_{\mathfrak{L}_{1}} \tilde{\delta}_{\mathfrak{L}_{2}} .
\end{aligned}
$$

After the correct statements of theorems are found, their proofs may be easily developed by induction on $k \geqslant 1$. To do this we can use the technique from [2]. The proofs given below is kindly provided by V. A. Fedchenko. They are simpler than the original proofs obtained by the first author and are based on the following two lemmas from [12].

Lemma 1. Let $f: V_{n} \rightarrow V_{m}, f=g \cdot h$, i.e. $f(x)=h(g(x))$ for all $x \in V_{n}$, where $g: V_{n} \rightarrow V_{t}$ and $h: V_{t} \rightarrow V_{m}, n, m, t \in \mathbb{N}$. Then for all $l^{\prime} \in V_{n}^{*}$, $l^{\prime \prime} \in V_{m}^{*}$ we have $\delta_{l^{\prime}, l^{\prime \prime}}^{f}=\sum_{l \in V_{t}^{*}} \delta_{l^{\prime}, l_{l, l^{\prime \prime}}^{g}}^{h}$.

Lemma 2. Let $f: V_{n} \rightarrow V_{m}, n, m \in \mathbb{N}$. For all $d^{\prime} \in V_{n}, d^{\prime \prime} \in V_{m}$ we have $p_{d^{\prime}, d^{\prime \prime}}^{f}=\frac{1}{2^{m}} \sum_{l^{\prime} \in V_{n}^{*}, l^{\prime \prime} \in V_{m}^{*}}(-1)^{d^{\prime} l^{\prime}+d^{\prime \prime} l^{\prime \prime}}\left(\delta_{l^{\prime}, l^{\prime \prime}}^{f}\right)^{2}$.

Proof of theorem 1. Let $k=1$. Using the notation of section 3 we get $b=a c_{02}+f_{1}\left(a c_{01}\right) c_{12}, C_{\mathcal{F}}=\left(\begin{array}{cc}c_{01} & c_{02} \\ 0 & c_{12}\end{array}\right)$,

$$
\mathfrak{W}_{\mathcal{F}}\left(L^{\prime}, L^{\prime \prime}\right)=\left\{\left(l, c_{12} L^{\prime \prime}\right) \mid l \in V_{n_{1}}^{*}: c_{01} l+c_{02} L^{\prime \prime}=L^{\prime}\right\} .
$$

Let $\widehat{F}: V_{N} \rightarrow V_{n_{1}}, \widehat{F}(a)=f_{1}\left(a c_{01}\right)$. Applying lemma 1 to $f=\widehat{F}$, using equation

$$
\mathbf{P}\left\{a L^{\prime}+b L^{\prime \prime}=0\right\}=\mathbf{P}\left\{a\left(L^{\prime}+c_{02} L^{\prime \prime}\right)+\widehat{F}(a) c_{12} L^{\prime \prime}=0\right\}
$$

we obtain $\delta_{L^{\prime}, L^{\prime \prime}}^{F}=\delta_{L^{\prime}+c_{02} L^{\prime \prime}, c_{12} L^{\prime \prime}}^{\widehat{F}}=\sum_{l \in V_{n_{1}}^{*}: L^{\prime}+c_{02} L^{\prime \prime}=c_{01} l} \delta_{l, c_{12} L^{\prime \prime}}^{f_{1}}$, this corresponds the statement of theorem 1.

Now suppose that $k>1$. We decompose the functional scheme $\mathcal{F}$ of $F: V_{N} \rightarrow V_{M}$ into schemes $\mathcal{F}_{1}$ and $\mathcal{F}_{2}$ for the mappings $F_{1}: V_{N} \rightarrow V_{K}$ and $F_{2}: V_{K} \rightarrow V_{M}, F=F_{1} \circ F_{2}, a \stackrel{F_{1}}{\longmapsto} z \stackrel{F_{2}}{\longmapsto} b, a \in V_{N}, z \in V_{K}, b \in V_{M}$. Let the elements $f_{1}, \ldots, f_{k_{1}}$ be included in the functional scheme $\mathcal{F}_{1}$, and the elements $f_{k_{1}+1}, \ldots, f_{k_{1}+k_{2}}$ be included in $\mathcal{F}_{2}$, where $k_{1}+k_{2}=k, k_{1}<k$, $k_{2}<k$. 
Now we show that there is a bijection $\iota$ between sets $\mathfrak{W}_{\mathcal{F}}\left(L^{\prime}, L^{\prime \prime}\right)$ and $\bigcup_{\Lambda \in V_{K}^{*}}\left(\mathfrak{W}_{\mathcal{F}_{1}}\left(L^{\prime}, \Lambda\right) \times \mathfrak{W}_{\mathcal{F}_{2}}\left(\Lambda, L^{\prime \prime}\right)\right)$. Suppose that $F_{1}(a)=z$,

$$
\begin{aligned}
x^{(1)}=\left(x_{1}, \ldots, x_{k_{1}}\right), & y^{(1)}=\left(y_{1}, \ldots, y_{k_{1}}\right), \\
x^{(2)}=\left(x_{k_{1}+1}, \ldots, x_{k_{1}+k_{2}}\right), & y^{(2)}=\left(y_{k_{1}+1}, \ldots, y_{k_{1}+k_{2}}\right) ;
\end{aligned}
$$

then $\left(a, y^{(1)}\right) C_{\mathcal{F}_{1}}=\left(x^{(1)}, z\right),\left(z, y^{(2)}\right) C_{\mathcal{F}_{2}}=\left(x^{(2)}, b\right)$ or, in more detail,

$$
\begin{array}{ll}
x^{(1)}=a C_{\mathcal{F}_{1}}^{(00)}+y^{(1)} C_{\mathcal{F}_{1}}^{(10)}, & z=a C_{\mathcal{F}_{1}}^{(01)}+y^{(1)} C_{\mathcal{F}_{1}}^{(11)}, \\
x^{(2)}=z C_{\mathcal{F}_{2}}^{(00)}+y^{(2)} C_{\mathcal{F}_{2}}^{(10)}, & b=z C_{\mathcal{F}_{2}}^{(01)}+y^{(2)} C_{\mathcal{F}_{2}}^{(11)} .
\end{array}
$$

From the last four equalities we get

$$
\begin{gathered}
x^{(1)}=a C_{\mathcal{F}_{1}}^{(00)}+y^{(1)} C_{\mathcal{F}_{1}}^{(10)}, \\
x^{(2)}=a C_{\mathcal{F}_{1}}^{(01)} C_{\mathcal{F}_{2}}^{(00)}+y^{(1)} C_{\mathcal{F}_{1}}^{(11)} C_{\mathcal{F}_{2}}^{(00)}+y^{(2)} C_{\mathcal{F}_{2}}^{(10)}, \\
b=a C_{\mathcal{F}_{1}}^{(01)} C_{\mathcal{F}_{2}}^{(01)}+y^{(1)} C_{\mathcal{F}_{1}}^{(11)} C_{\mathcal{F}_{2}}^{(01)}+y^{(2)} C_{\mathcal{F}_{2}}^{(11)} .
\end{gathered}
$$

Combining last three equalities and equality $\left(a, y^{(1)}, y^{(2)}\right) C_{\mathcal{F}}=\left(x^{(1)}, x^{(2)}, b\right)$ we obtain that $C_{\mathcal{F}}$ is represented by $(3 \times 3)$-block matrix with blocks $C_{\mathcal{F}_{s}}^{(i j)}$, $i, j=0,1, s=1,2$. By the inductive assumption using lemma 1 and bijection $\iota$ we finally get

$$
\begin{aligned}
& \delta_{L^{\prime}, L^{\prime \prime}}^{F}=\sum_{\Lambda \in V_{K}^{*}}\left(\delta_{L^{\prime}, \Lambda}^{F_{1}} \cdot \delta_{\Lambda, L^{\prime \prime}}^{F_{2}}\right) \\
& =\sum_{\Lambda \in V_{K}^{*}}\left[\left(\sum_{\mathfrak{L}_{1} \in \mathfrak{W}_{\mathcal{F}_{1}}\left(L^{\prime}, \Lambda\right)} \widetilde{\delta}_{\mathfrak{L}_{1}}\right) \cdot\left(\sum_{\mathfrak{L}_{2} \in \mathfrak{W}_{\mathcal{F}_{2}}\left(\Lambda, L^{\prime \prime}\right)} \widetilde{\delta}_{\mathfrak{L}_{2}}\right)\right] \\
& =\sum_{\Lambda \in V_{K}^{*}} \sum_{\substack{\mathfrak{L}_{1} \in \mathfrak{W}_{\mathcal{F}_{1}}\left(L^{\prime}, \Lambda\right) \\
\mathfrak{L}_{2} \in \mathfrak{W}_{\mathcal{F}_{2}}\left(\Lambda, L^{\prime \prime}\right)}}\left(\widetilde{\delta}_{\mathfrak{L}_{1}} \cdot \widetilde{\delta}_{\mathfrak{L}_{2}}\right)=\sum_{\substack{\mathfrak{L} \in \mathfrak{W}_{\mathcal{F}}\left(L^{\prime}, L^{\prime \prime}\right)\\
}} \widetilde{\delta}_{\mathfrak{L}} .
\end{aligned}
$$

This completes the proof of theorem 1 .

Proof of theorem 2. By lemma 2 and theorem 1 we get that theorem 2 is equivalent to the equality

$$
\sum_{\mathfrak{D} \in W_{\mathcal{F}}\left(D^{\prime}, D^{\prime \prime}\right)} \widetilde{p}_{\mathfrak{D}}=\frac{1}{2^{M}} \sum_{\left(L^{\prime}, L^{\prime \prime}\right) \in V_{N}^{*} \times V_{M}^{*}}(-1)^{D^{\prime} L^{\prime}+D^{\prime \prime} L^{\prime \prime}} \sum_{\mathfrak{L} \in \mathfrak{W}_{\mathcal{F}}\left(L^{\prime}, L^{\prime \prime}\right)} \widetilde{\delta}_{\mathfrak{L}}^{2} .
$$

The equality (4) is proved by induction on $k$. Let $C_{1}$ and $C_{2}$ be Boolean matrices of sizes $N \times N_{1}$ and $N \times M$ respectively, and $F(a)=G\left(a C_{1}\right)+a C_{2}$. 
Suppose the mapping $G: V_{N_{1}} \rightarrow V_{M}$ is specified using the functional scheme $\mathcal{G}$. Taking into account the representation of the matrix $C_{\mathcal{F}}$ using matrix $C_{\mathcal{G}}$, we obtain

$$
\begin{gathered}
W_{\mathcal{F}}\left(D^{\prime}, D^{\prime \prime}\right)=W_{\mathcal{G}}\left(D^{\prime} C_{1}, D^{\prime \prime}+D^{\prime} C_{2}\right), \\
\mathfrak{W}_{\mathcal{F}}\left(L^{\prime}, L^{\prime \prime}\right)=\cup_{L \in V_{N_{1}}^{*}}: C_{1} L=L^{\prime}+C_{2} L^{\prime \prime} \mathfrak{W}_{\mathcal{G}}\left(L, L^{\prime \prime}\right) .
\end{gathered}
$$

This implies that for $k=1$ it is sufficient to prove the equality (4) for $F(a)=b=f_{1}(a) c_{12}$. But in this case (for this $F$ ) theorem 2 is obviously true since the set $\mathfrak{W}_{\mathcal{F}}\left(L^{\prime}, L^{\prime \prime}\right)$ contains the unique local linear relation $\left(L^{\prime}, c_{12} L^{\prime \prime}\right)$ and $W_{\mathcal{F}}\left(D^{\prime}, D^{\prime \prime}\right)=\left\{\left(D^{\prime}, D\right) \mid D c_{12}=D^{\prime \prime}\right\}$. Indeed, we get

$$
\frac{1}{2^{M}} \sum_{\left(L^{\prime}, L^{\prime \prime}\right) \in V_{N}^{*} \times V_{M}^{*}}(-1)^{D^{\prime} L^{\prime}+D^{\prime \prime} L^{\prime \prime}} \sum_{\substack{\mathfrak{L}_{1}, \mathfrak{L}_{2} \in \mathfrak{2}_{\mathcal{F}}\left(L^{\prime}, L^{\prime \prime}\right): \\ \mathfrak{L}_{1} \neq \mathfrak{L}_{2}}} \tilde{\delta}_{\mathfrak{L}_{1}} \tilde{\delta}_{\mathfrak{L}_{2}}=0,
$$

and theorem 2 follows from the total probability law. The inductive transition is the same as in theorem 1 . This completes the proof.

Note that theorem 1 have been proved earlier in special cases (see $[12,14])$.

In view of these theorems, the search for linear and differential relations (see sections 3 and 4) may be performed by maximizing absolute values of individual summands $\left|\tilde{\delta}_{\mathfrak{L}}\right|$ and $\tilde{p}_{\mathfrak{D}}$ in formulas for exact values $\delta_{L^{\prime}, L^{\prime \prime}}$ and $p_{D^{\prime}, D^{\prime \prime}}$. But these maximizations should be carried out only for all $\mathfrak{L} \in \mathfrak{W}^{(0)} \backslash\{0\}$ and for all $\mathfrak{D} \in W^{(0)} \backslash\{0\}$. This fact is an additional motivation for the choice $\tilde{\delta}_{\mathfrak{L}}$ and $\tilde{p}_{\mathfrak{D}}$ as rough approximations for $\delta_{L^{\prime}, L^{\prime \prime}}$ and $p_{D^{\prime}, D^{\prime \prime}}$.

Also in view of theorems 1 and 2 we can see the disadvantages of the technique of linear and differential cryptanalysis described above. Here are the main disadvantages.

1. We are searching for relations that we can find, not for the best ones.

2. General results on the degree of approximations for linear and differential advantages are not available.

3. If we focus on problems of finding $\tilde{\delta}=\max _{\mathfrak{L} \in \mathfrak{W J}^{(0)} \backslash\{0\}}\left|\tilde{\delta}_{\mathfrak{L}}\right|$ and $\tilde{p}=$ $\max _{\mathfrak{D} \in W^{(0)} \backslash\{0\}} \tilde{p}_{\mathfrak{D}}$, then we may leave the domains containing the values $\delta=\max _{L^{\prime} \in V_{N}^{*}, L^{\prime \prime} \in V_{M}^{*} \backslash\{0\}}\left|\delta_{L^{\prime}, L^{\prime \prime}}\right|$ and $p=\max _{D^{\prime} \in V_{N} \backslash\{0\}, D^{\prime \prime} \in V_{M}} p_{D^{\prime}, D^{\prime \prime}}$.

Let's explain the last item. From theorems 1 and 2 it follows that problems of finding $\tilde{\delta}$ and $\delta$, as well as $\tilde{p}$ and $p$, are different optimization problems. If $\tilde{\delta}=\left|\tilde{\delta}_{\mathfrak{L}_{\mathfrak{o}}}\right|, \delta=\left|\delta_{L_{0}^{\prime}, L_{0}^{\prime \prime}}\right|$, then it is not necessary that 
$L_{0}^{\prime}=L_{\mathfrak{L}_{\mathrm{o}}}^{\prime}, L_{0}^{\prime \prime}=L_{\mathfrak{L}_{\mathrm{o}}}^{\prime \prime}$. For example, for DES with 14 iterations the pair $\left(L_{0}^{\prime}, L_{0}^{\prime \prime}\right) \in\left(V_{64}^{*} \backslash\{0\}\right)^{2}$ is not known, although $L_{\mathfrak{L}_{\mathfrak{o}}}^{\prime}, L_{\mathfrak{L}_{\circ}}^{\prime \prime}$ are known. For differential relations the situation is similar.

We have to put up with these disadvantages because the problems of maximizing the values $\left|\delta_{L^{\prime}, L^{\prime \prime}}\right|$ and $p_{D^{\prime}, D^{\prime \prime}}$ is much more difficult then the problems of maximizing the values $\left|\tilde{\delta}_{\mathfrak{L}}\right|$ and $\tilde{p}_{\mathfrak{D}}$ as it may be seen from theorems 1 and 2 .

Of course, the assumptions on the uniformity of random variables $x_{i} \in V_{n_{i}}, i=1, \ldots, k$, and on the independence of events in motivations formulated above are not satisfied as a rule. Nevertheless, SOMETIMES we have $\tilde{\delta} \approx \delta, \tilde{p} \approx p$, for example, $3^{-1} \leqslant \tilde{\delta} /|\delta| \leqslant 3,10^{-1} \leqslant \tilde{p} / p \leqslant 10$. We can verify this by computing empirical estimates of $\delta^{*}$ for $\delta, p^{*}$ for $p$ and appropriate confidence intervals. High precision estimates of $\delta$ and $p$ are not required. (If the empirical estimates do not correspond the accuracy of measurements, this may lead to the study of phenomena that are not related to the nature of the measured quantity.) The term SOMETIMES refers to a sets of all possible cipher transformations and chains of conformal local probabilistic relations obtained by the cryptanalyst. Functions $\operatorname{SOMETIMES}(F, \mathcal{F}, \mathfrak{L})$ and $\operatorname{SOMETIMES}(F, \mathcal{F}, \mathfrak{D})$ have two possible values: fair $\approx$ and unfair $\approx$.

If the values $\left|\tilde{\delta}_{\mathfrak{L}}\right|, \tilde{p}_{\mathfrak{D}}$ are small, then the verification of the approximate equalities $\tilde{\delta}_{\mathfrak{L}} \approx \delta_{L_{\mathfrak{L}}^{\prime}, L_{\mathfrak{L}}^{\prime \prime}}, \tilde{p}_{\mathfrak{D}} \approx p_{D_{\mathfrak{O}}^{\prime}, D_{\mathfrak{D}}^{\prime \prime}}$ is complicated due to limited computing resources. In this case we have to consider a close analogues of the investigated cipher. According to the authors of this paper, the best analogues are those ciphers that have the same linear medium, even with the same number of iterations. In this case the equalities in theorems 1 and 2 have the same form. A verification should be carried out for as many situations as possible. For this purpose for the fixed $n_{i}$ and $m_{i}$ the functional elements $\tilde{f}_{i}: V_{n_{i}} \rightarrow V_{m_{i}}, i=1, \ldots, k$, should be varied so as to increase $\left|\tilde{\delta}_{\mathfrak{L}}\right|, \tilde{p}_{\mathfrak{D}}$. And for some indices $i \in\{1, \ldots, k\}$ even the inequalities $\left|\delta_{l_{i}^{\prime}, l_{i}^{\prime \prime}}^{\tilde{f}_{i}}\right|<\left|\delta_{l_{i}^{\prime}, l_{i}^{\prime \prime}}^{f_{i}}\right|, p_{d_{i}^{\prime}, d_{i}^{\prime \prime}}^{\tilde{f}_{i}}<p_{d_{i}^{\prime}, d_{i}^{\prime \prime}}^{f_{i}}$ may be achieved.

The values $\tilde{\delta}$ and $\tilde{p}$ have preferences and drawbacks, but they are additional characteristics which may be more important then $\delta$ and $p$. And it doesn't even matter that they are close to or far from $\delta$ and $p$. The values $\delta$ and $p$, as a rule, do not lend themselves (unlike $\tilde{\delta}$ and $\tilde{p}$ ) to any reasonable estimates in the case of real cipher conversions.

Although the differential cryptanalysis is applied more frequently then linear cryptanalysis, in some situations the linear cryptanalysis may be 
preferable, for example, when $1 / \delta^{2} \ll 1 / p$. But even if $1 / \delta^{2} \approx 1 / p$, then the linear cryptanalysis may be preferable, because a linear attack is a plaintext only attack, while a differential attack is a chosen plaintext attack (or a chosen ciphertext attack).

\section{The duality of differential and linear cryptanalysis}

The comparison of sections 3 and 4 shows that the problems of finding linear and differential relations are identical. It is not accidental [2]. The equality (2) follows from $\left(l_{1}^{\prime T}, \ldots, l_{k}^{\prime T}, L^{\prime \prime T}\right) C^{T}=\left(L^{\prime T}, l_{1}^{\prime \prime T}, \ldots, l_{k}^{\prime \prime T}\right)$ by transposition or from equality $\left(L^{\prime \prime T}, l_{k}^{\prime T}, \ldots, l_{1}^{\prime T}\right) C^{*}=\left(l_{k}^{\prime \prime T}, \ldots, l_{1}^{\prime \prime T}, L^{\prime T}\right)$, where the matrix $C^{*}=\left\|c_{i j}^{*}\right\|, i=0,1, \ldots, k, j=1, \ldots, k, k+1$, is obtained from the matrix $C^{T}$ by centrally symmetric permutation of blocks: $c_{i j}^{*}=c_{k+1-j, k+1-i}^{T}$.

Consider the fresh concept of a dual functional scheme $\mathcal{F}^{*}$ with the linear medium $C^{*}$ and with the tables $\left\|\delta_{l^{\prime}, l^{\prime \prime}}^{f_{i}^{*}}\right\|, l^{\prime} \in V_{m_{k+1-i}}^{*}, l^{\prime \prime} \in V_{n_{k+1-i}}^{*},\left\|p_{d^{\prime}, d^{\prime \prime}}^{f_{*}^{*}}\right\|$, $d^{\prime} \in V_{m_{k+1-i}}, d^{\prime \prime} \in V_{n_{k+1-i}}, i=1, \ldots, k$, instead of functions $f_{i}^{*}: V_{m_{i}} \rightarrow V_{n_{i}}$, $\delta_{l^{\prime}, l^{\prime \prime}}^{f_{i}^{*}}=p_{l^{\prime \prime T}, l^{\prime T}}^{f_{i}}, p_{d^{\prime}, d^{\prime \prime}}^{f_{i}^{*}}=\delta_{d^{\prime \prime T}, d^{\prime T}}^{f_{i}}$. In sections 3 and 4 knowledge of the elements of the tables is sufficient; function $f_{i}$ values are not required.

Thus, the problem of finding probabilistic differential relations of the cipher $F$ defined by the functional scheme $\mathcal{F}$ with the linear medium $C$ and local nonlinear functions $f_{i}: V_{n_{i}} \rightarrow V_{m_{i}}, i=1, \ldots, k$, is equivalent to the problem of finding probabilistic linear relations for a dual functional scheme $\mathcal{F}^{*}$. Similarly, the problem of finding probabilistic linear relations of the cipher $F$ is equivalent to the problem of finding probabilistic differential relations for a functional scheme $\mathcal{F}^{*}$.

\section{Universal functional scheme}

Let $F: V_{N} \rightarrow V_{M}$ and $\widetilde{F}: V_{N} \rightarrow V_{M}$ be two mappings defined by the functional schemes $\mathcal{F}$ and $\widetilde{\mathcal{F}}$ respectively that have the same linear media, i.e. $\widetilde{k}=k, \widetilde{n}_{i}=n_{i}, \widetilde{m}_{i}=m_{i}, i=1, \ldots, k, \widetilde{C}=C$. Further, let for functional elements $f_{i}: V_{n_{i}} \rightarrow V_{m_{i}}, \widetilde{f}_{i}: V_{n_{i}} \rightarrow V_{m_{i}}$ we have

$$
\left|\delta_{l_{i}^{\prime}, l_{i}^{\prime \prime}}^{f_{i}}\right|=\left|\delta_{l_{i}^{\prime}, l_{i}^{\prime \prime}}^{\widetilde{f_{i}}}\right|, \quad p_{d_{i}^{\prime}, d_{i}^{\prime \prime}}^{f_{i}}=p_{d_{i}^{\prime}, d_{i}^{\prime \prime}}^{\widetilde{f_{i}}}
$$

for all $l_{i}^{\prime} \in V_{n_{i}}^{*}, l_{i}^{\prime \prime} \in V_{m_{i}}^{*}, d_{i}^{\prime} \in V_{n_{i}}, d_{i}^{\prime \prime} \in V_{m_{i}}, i=1, \ldots, k$. Then the conclusions of sections 3 and 4 will be the same for $F$ and $\widetilde{F}$. 
Below we construct a family of functional schemes with a fixed linear medium $C$ that implement any map from $V_{N}$ to $V_{M}$. Moreover for any pair of functional schemes of this family the equalities (5) are valid. Then the conclusions of sections 3 and 4 will be the same for all mappings from $V_{N}$ to $V_{M}$.

The universal functional scheme (UFS) is the family of $2^{M 2^{N}}$ functional schemes parameterized by a vector $\bar{\varepsilon}=\left(\varepsilon_{1}, \ldots, \varepsilon_{M 2^{N}}\right) \in V_{M 2^{N}}$. These functional schemes define all $2^{M 2^{N}}$ mappings $F_{\bar{\varepsilon}}: V_{N} \rightarrow V_{M}, \bar{\varepsilon} \in V_{M 2^{N}}$. A scheme $\mathfrak{f}_{\varepsilon}, \varepsilon \in G F(2)$, with two inputs and one output will be the main structural element of UFS. It consists of two functional elements $f_{1, \varepsilon}: V_{2} \rightarrow G F(2)$ and $f_{2}: V_{2} \rightarrow G F(2)$ defined by formulas $f_{1, \varepsilon}\left(x_{0}, x_{1}\right)=$ $\left(x_{0}+\varepsilon\right)\left(x_{1}+1+\varepsilon\right), f_{2}\left(x_{0}, x_{1}\right)=x_{0} x_{1}$. By definition, we put

$$
\mathfrak{f}_{\varepsilon}\left(x_{0}, x_{1}\right)=f_{1, \varepsilon}\left(x_{0}, x_{1}\right)+f_{2}\left(x_{0}, x_{1}\right)=(1+\varepsilon) x_{0}+\varepsilon x_{1} .
$$

For all $l^{\prime} \in V_{2}^{*}, l^{\prime \prime} \in V_{1}^{*}$ the values $\left|\delta_{l^{\prime}, l^{\prime \prime}}^{f_{\varepsilon}}\right|$ do not depend on $\varepsilon$. Also for all $d^{\prime} \in V_{2}, d^{\prime \prime} \in V_{1}$ the values $p_{d^{\prime}, d^{\prime \prime}}^{f_{\varepsilon}}$ do not depend on $\varepsilon$.

The UFS with $N$ binary inputs $\left(a_{1}, a_{2}, \ldots, a_{N}\right) \in V_{N}$ has a first part that does not depend on $\bar{\varepsilon} \in V_{M 2^{N}}$. The first part of UFS produces all monomials

$$
1, a_{1}, \ldots, a_{N}, a_{1} a_{2}, \ldots, a_{i} a_{j}, \ldots, a_{N-1} a_{N}, \ldots, a_{1} \ldots a_{N} .
$$

No more than $2^{N}$ functional elements $f_{2}$ and one functional element $g: G F(2) \rightarrow G F(2), g(x) \equiv 1$, are required to produce all monomials (6). The second part of UFS depends on $\bar{\varepsilon} \in V_{M 2^{N}}$ and contains $M 2^{N}$ schemes $\mathfrak{f}_{\varepsilon}$ divided into $M$ classes. Each class corresponds to one output of UFS and contains $2^{N}$ schemes $\mathfrak{f}_{\varepsilon}$. Each component of the vector $\bar{\varepsilon}=\left(\varepsilon_{1}, \ldots, \varepsilon_{M 2^{N}}\right) \in V_{M 2^{N}}$ corresponds to its own functional scheme $\mathfrak{f}_{\varepsilon}$. All monomials (6) are used as inputs $x_{1}$ of functional schemes $\mathfrak{f}_{\varepsilon}$ from every class. The value $0=1+1$ is used as input $x_{0}$ for each functional scheme $\mathfrak{f}_{\varepsilon}$. Each output of UFS is a sum of outputs of all $2^{N}$ schemes $\mathfrak{f}_{\varepsilon}$ from the corresponding class.

We may obtain any function $V_{N} \rightarrow V_{M}$ by selecting the vector $\bar{\varepsilon}$ for each of $M 2^{N}$ schemes. Each function corresponds to its own scheme. Each of the $2^{M 2^{N}}$ functional schemes has the same linear medium. For the corresponding functional elements, including the elements $\left(x_{0}+\varepsilon\right)\left(x_{1}+1+\varepsilon\right)$ with $\varepsilon=0$ and $\varepsilon=1$, the equalities (5) are valid. If we use these functional schemes and follow the recommendations from sections 3 and 4, then for all functions $V_{N} \rightarrow V_{M}$ we obtain the same "best" probabilistic linear relation 
and the same "best" differential relation. This is an extreme example of the first disadvantage from section 5 . The universal functional scheme allows us to obtain exotic examples of relations both between $\tilde{\delta}$ and $\delta$ and between $\tilde{p}$ and $p$.

\section{Role of cryptographic keys in linear and differential cryptanalysis}

As a rule, many authors obtain probabilistic linear and differential relations and estimate their advantages directly for functions $\Phi: V_{N} \times V_{K} \rightarrow$ $V_{M},(a, X) \mapsto b=\Phi(a, X)$, where $X \in V_{K}$ is a cryptographic key. They often use various kinds of probabilistic models for the dependence of iteration keys on $X$ (in addition to the probability distributions on $V_{N} \times V_{K}$ ).

The authors of this paper are deeply convinced of the following. Probabilistic relations used to determine the key $Z$ that is applied before the mapping $\Phi$. Probabilistic relations should be constructed for functions $F_{X}: a \mapsto b=\Phi(a, X)$ in accordance with sections 3 and 4 for each key $X$ separately. The values of the argument $a \in V_{N}$ of the mapping $F_{X}$ are generated from plain text and the key $Z, a=\varphi(\tilde{a}, Z)$, where $\tilde{a}$ is the plain text, $\varphi$ - some known famous function. For some keys a nontrivial probabilistic relation may not be found for various reasons. But sometimes the probabilistic relations based on the same chains may be useful for wide classes of keys $X$. In such situations the effective guessing of the key $Z$ may be expected.

Our point of view is difficult to challenge, for example, for a cipher such that on every iteration all components of current block are permuted by some permutation depending on the key $X$. The complexity, the probability of success, the number of plaintext/ciphertext pairs needed to determine the key $Z$ are random values which depend on the random $X \in V_{K}$. Recall that $X$ is not the key to find.

\section{The importance of linear and differential cryptanalysis for cipher design}

We may consider the problem of minimizing parameters $\tilde{\delta}$ and $\tilde{p}$ in the process of cipher design as a formalization of a rule that ciphers should be as nonlinear as possible. This is achieved by decreasing the absolute values of linear and differential advantages and by increasing the degrees of diffusion $\theta$ and $\theta^{\prime}$ of the linear medium. The latter explains the transition 
from SP-networks (in which Shannon's idea of confusion and diffusion were realized in the second half of the last century [3]) to XSL-ciphers. A new cipher design technique has appeared. This technique is based on guaranteeing high diffusion degrees $\theta$ and $\theta^{\prime}$ of cipher's linear medium. Cipher design starts with the construction of a general structure, i.e. a linear medium. The nonlinear mappings are not specified at the preliminary stage of cipher design. They are considered in general form. The diffusion degrees of linear medium are evaluated before the specification of nonlinear mappings. For higher diffusion degrees it is easier to guarantee the security of a cipher with respect to linear, differential and other techniques of cryptanalysis by the careful selection of nonlinear mappings included in the functional scheme. The degrees of diffusion $\theta$ and $\theta^{\prime}$ of the linear medium (along with the number of iterations and the cardinality of the key set) are unusual parameters that are easy to estimate and at the same time allow to make conclusions on the security level of the cipher.

The diffusion degrees $\theta$ and $\theta^{\prime}$ of $X S L$-cipher with two iterations are the coefficients of diffusion $\rho_{\Lambda}$ and $\rho_{\Lambda}^{\prime}$ for separate nonsingular linear transformation $\Lambda \in G L(n, 2)$ [2]. Similar diffusion degrees of linear medium related to $s$-dimensional linear cryptanalysis are also important. They are more valuable diffusion parameters of permutation matrices used in $S P$-networks then $\theta$ and $\theta^{\prime}[4]$.

The papers [5-13] give a different understanding of the methods of constructing probabilistic linear and differential relations without separation of the linear medium of the cipher. The linear medium provides a more rigorous theoretical justification of the methods.

The authors thank V. A. Fedchenko for the proofs of theorems 1 and 2 and also the anonymous reviewer for careful reading of the paper and very useful comments. The authors are especially grateful to A.M. Zubkov for his attention to problems considered.

\section{References}

[1] Erokhin A. V., Malyshev F. M., Trishin A. E., "Multidimentional linear method and diffusion characteristics of linear medium of ciphering transform", Matematicheskie Voprosy Kriptografii, 8:4 (2017), 29-62.

[2] Malyshev F. M., "The duality of differential and linear methods in cryptography", Matematicheskie Voprosy Kriptografi, 5:3 (2014), 35-47.

[3] Massey J. L., "An introduction to contemporary cryptology", Proc. IEEE, 76:5 (1988), $533-549$. 
[4] Malyshev F. M., Trifonov D. I., "Diffusion properties of XSLP-ciphers", Matematicheskie Voprosy Kriptografii, 7:3 (2016), 47-60.

[5] Biham E., Shamir A., "Differential cryptanalysis of DES-like cryptosystems", CRYPTO'90, Lect. Notes Comput. Sci., 537, 1991, 2-21.

[6] Biham E., Shamir A., "Differential cryptanalysis of DES-like cryptosystems", J. Cryptology, 4:1 (1991), 3-72.

[7] Matsui M., "Linear cryptanalysis method for DES Cipher", EUROCRYPT'93, Lect. Notes Comput. Sci., 765, 1994, 386-397.

[8] Matsui M., "The first experimental cryptanalysis of the Data Encryption Standard", CRYPTO'94, Lect. Notes Comput. Sci., 839, 1994, 1-11.

[9] Biham E., "On Matsui's linear cryptanalysis", EUROCRYPT'94, Lect. Notes Comput. Sci., 950, 1995, 341-355.

[10] Matsui M., "On correlation between the order of S-boxes and the strength of DES", EUROCRYPT'94, Lect. Notes Comput. Sci., 950, 1995, 366-375.

[11] Nyberg K., "Linear approximation of block ciphers", EUROCRYPT'94, Lect. Notes Comput. Sci., 950, 1995, 439-444.

[12] Daemen J., Govaerts R., Vandewalle J., "Correlation matrices", FSE'94, Lect. Notes Comput. Sci., 1008, 1995, 275-285.

[13] Borst J., Preneel B., Vandewalle J., "Linear cryptanalysis of RC5 and RC6", FSE'99, Lect. Notes Comput. Sci., 1636, 1999, 16-30.

[14] Daemen J., Rijmen V., The Design of Rijndael: AES - The Advanced Encryption Standard, Springer-Verlag, Berlin-Heidelberg, 2002, xvii+238 pp. 